\title{
Use of Esophageal Stents After Anastomotic Leakage in Surgery for Gastric Adenocarcinoma Case Report and Review of the Literature
}

\author{
Mendoza-Moreno F*1, Díez-Gago MR $^{2}$, Mínguez-García J ${ }^{1}$, Enjuto-Martínez DT ${ }^{1}$,Tallón-Iglesias B $^{1}$, Solana-Maoño M ${ }^{1}$ \\ and Argüello-de-Andrés JM ${ }^{1}$ \\ ${ }^{1}$ Department of General and Digestive Surgery,Sanitas La Moraleja Teaching Hospital, Spain \\ ${ }^{2}$ Department of Emergency, Príncipe de Asturias Teaching Hospital, Spain
}

Received: 阱 July 4, 2018; Published: 制 July 12, 2018

*Corresponding author: Fernando Mendoza-Moreno, Department of General and Digestive Surgery, Sanitas La Moraleja Teaching Hospital, Madrid, Spain

\begin{abstract}
Introduction: Radical gastrectomy is the treatment of choice for the treatment of gastric cancer located in the upper third of stomach or in case of diffuse histology or cells in a signet ring. The worst complication after a radical gastrectomy is the leakage of the esophago-jejunal anastomosis, since it considerably increases the morbidity and mortality of the patient.

Case Report: Wedescribe our experience after performing a radical gastrectomy for gastric adenocarcinoma in a patient who developed a leakage of the esophago-jejunal anastomosis in the postoperative period. Although he was reoperated, performing reinforcement of the anastomosis and making a feeding jejunostomy, the dehiscence progressed in the following days until it became almost complete. Then, we proceeded to place a digestive endoprosthesis through gastroscopy with good results, allowing the entire defect to heal and being able to be removed without incidents after 8 weeks.

Discussion: There are several alternatives after a leakage of the esophago-jejunal anastomosis. Surgical options include reinforcement of the defect, resection and new anastomosis and esophageal exclusion. However, these options increase the morbidity and mortality of the patient and may require a second intervention in a second time to re-establish the transit. In recent years, the use of digestive stents placed by endoscopy in patients with anastomotic leakage after gastrectomy has proved to be a valid treatment option in selected patients.
\end{abstract}

Keywords: Stents; Endoscopy; Radical Gastrectomy; Anastomotic Leakage; Self Expandable Metal Stent

\section{Introduction}

Gastric cancer continues to be one of the malignant neoplasms of the gastrointestinal tract that causes the greatest number of cancer deaths in our days [1]. In patients with gastric cancer in the upper third or whose biopsy has confirmed the presence of a diffuse type adenocarcinoma or signet ring cells, a radical gastrectomy is the treatment of choice $[2,3]$. However, the dehiscence of esophagojejunal anastomosis after gastrectomy for cancer is between 3-20\% with a mortality of up to $60 \%$, greater after surgical reoperation than by endoscopic management $[4,5]$. The leakage of this anastomosis supposes an increase of the morbidity, since it is a delay in the beginning of the enteral nutrition increasing the hospital stay. In addition, its management is a challenge for the surgeon, since the surgical options range from reinforcing or redoing the anastomosis to performing an esophageal diversion. In recent years, the use of stents placed by endoscopy have been shown to reduce morbidity and mortality in selected patients, becoming a treatment option in the management of esophageal fistula.
Next, we describe the case of a patient who was operated for gastric adenocarcinoma, performing a radical gastrectomy, which presented as acomplication an esophago-jejunal fistula of practically all the circumference resolved by placement of an esophageal stent.

\section{Case Report}

A 76-year-old-man entered our center to perform a radical gastrectomy for cardia adenocarcinoma. His medical history was significant for hypertension, atrial fibrillation and chronic obstructive pulmonary disease. The patient reported a history of occasional dysphagia and epigastralgia of months of evolution. The gastroscopy observed an infiltrating lesion at the cardia whose biopsy was positive for intestinal type gastric adenocarcinoma. The extension study was performed by CT scan confirming a thickening at the level of the esophagogastric junction without the presence of pathological adenopathies at the mediastinal, thoracic or abdominal 
levels. The patient underwent a radical gastrectomy and type D1 lymphadenectomy with cholecystectomy and reconstruction of the transit according to Y-Roux. The esophageal-jejunal anastomosis was performed using a circular stapler (EEA No. 25 Fr) while the intestinal anastomosis was performed manually. No signs of peritoneal carcinomatosis were observed.

After surgery, the patient was admitted to the Intensive Care Unit (ICU) for monitoring and surveillance during the first 24 hours. On the sixth postoperative day, the patient began with fever and tachypnea accompanied by a purulent discharge from one of the abdominal drainages. A CT scan was performed with oral contrast, which showed esophageal-jejunal anastomotic leakage (Figure1). It was decided to perform urgent surgical intervention. After the opening of the laparotomy, minimal dehiscence of the right lateral posterolateral area of the esophago-jejunal anastomosis was observed. The duodenal stump and the intestinal anastomosis were intact. Reinforcement of the anastomosis was performed, tutoring of the anastomosis by a nasogastric tube, washing of the abdominal cavity and placement of drains near the reinforced anastomosis. A jejunostomy was made with the intention of starting enteral nutrition in the postoperative period. On his return from the operating room to the ICU, developing a situation of significant inflammatory response syndrome, with tachycardia, fever and increased acute phase reactants. The following days presented clinical improvement but with persistence of fever.

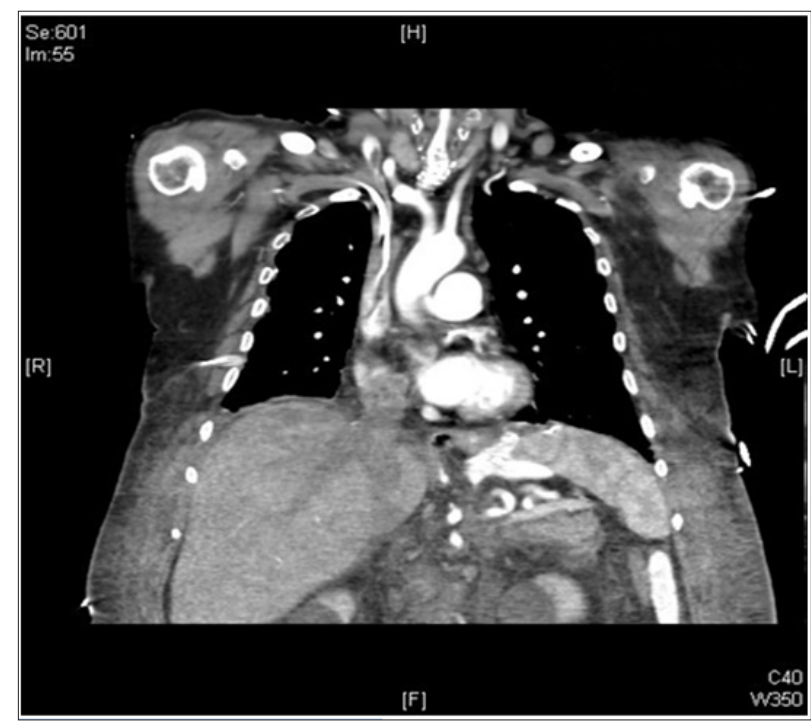

Figure 1: CT scan shows the leakage of oral contrast.

CT scan was performed that observed almost complete dehiscence of the esophago-jejunal anastomosis. Given the delicate general condition of our patient (requiring respiratory support through orotracheal intubation) we decided to place a digestive stent through endoscopy. Gastroscopy confirmed the progression of the dehiscence of more than $80 \%$ of the circumference of the esophago-jejunal anastomosis (Figure2). A complete coated stent (Hanarostent 20x130 mm) was placed in the proximal esophagus using clips (Figure3). After the placement of the stent, the patient showed a slow but progressive clinical improvement, remaining afebrile and without evidence of progression of sepsis. The main problem during his stay in the ICU was the respiratory situation, presenting data on respiratory distress associated with "rigid lung" secondary to the polyneuropathy of a critical patient he presented. After 2 months of stay in the Intensive Care Unit, the patient presented a subjective clinical improvement in the following days. There, the stent was removed after 8 weeks of placement, proving the absence of a residual fistula and initiating oral nutrition (Figure4). The jejunostomy catheter was removed without incident.

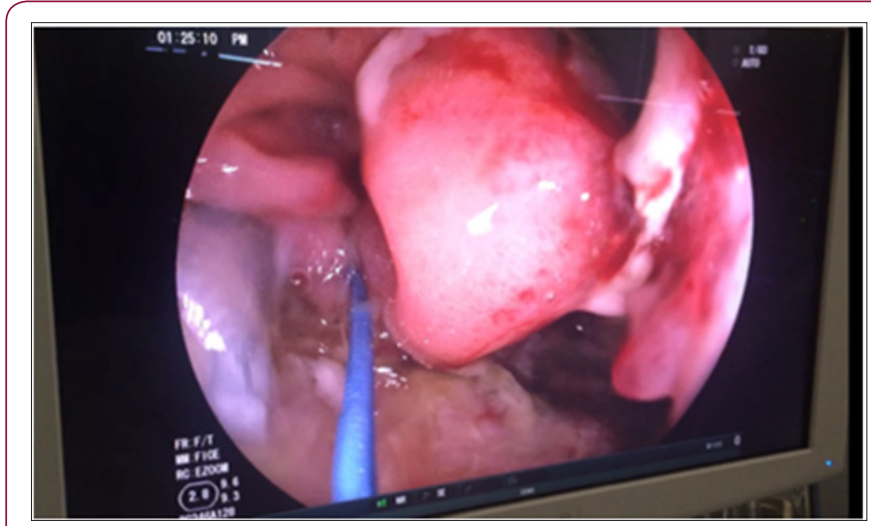

Figure 2: Anastomotic leakage of $80 \%$ of circumference showed in endoscopy.

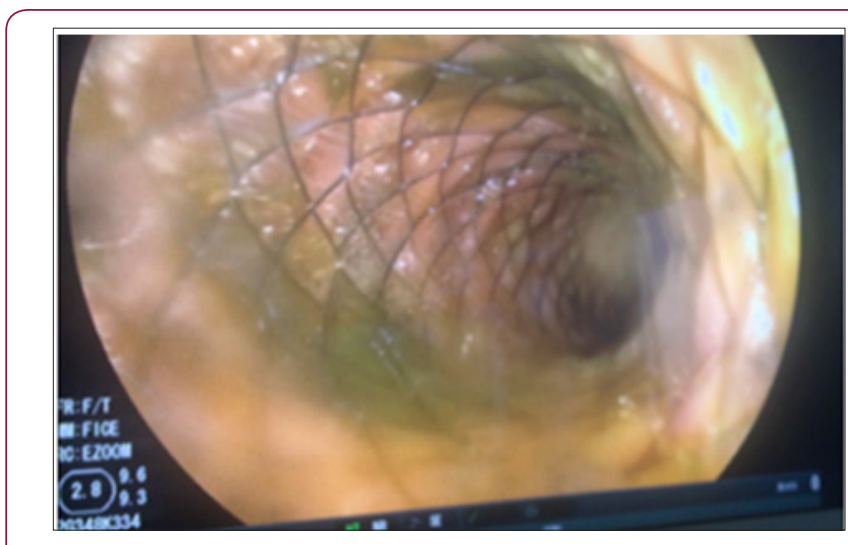

Figure 3: Self-expandable metal stent covering the anastomotic leakage.

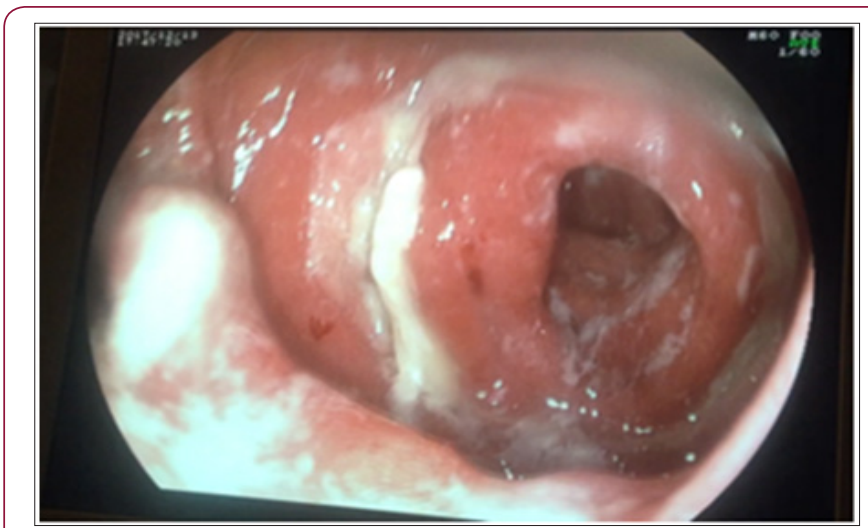

Figure 4: Anastomotic leakage after removing esophageal stent.

After 30 more days of hospitalization and thanks to the work of the rehabilitation service, the patient was discharged. It was not 
necessary to place any feeding tube for enteral nutrition so, since the removal of the prosthesis, the patient presented good tolerance progressively orally. The histopathological result confirmed the presence of a moderately differentiated intestinal adenocarcinoma with presence of vascular and perineural infiltration without involvement of surgical margins. None of the 30 resected nodes presented neoplastic infiltration.

\section{Discussion}

Anastomotic leakage in gastric esophageal surgery comprises rates of $10-25 \%$ at the cervical level and up to $20 \%$ in the thorax and abdomen, increasing the mortality associated with surgery up to 3 times more $[2,6]$.

According to the different authors, the dehiscence of the esophago-jejunal anastomosis is 3-20\% with a mortality of $9-64 \%$ $[2,7]$. The rate of anastomotic dehiscence is greater in a radical gastrectomy than in a subtotal gastrectomy [3]. Among the risk factors of the leakage of the anastomosis have been described related to the patient (hypertension, ischemic heart disease, previous lung disease, chronic kidney failure, diabetes mellitus or a visceral fat area index) as well as those related to the surgical technique ( surgical time, bleeding, use of circular stapler ...) [2,7]. Other factors such as the performance of robot-assisted gastrectomy have not shown a higher rate of esophageal fistula while for authors as Persson, smoking and advanced age are statistically significant risk factors related to the failure of the anastomosis $[1,8]$.

Dehiscence usually occurs between the fifth and the eighth postoperative day. The most important prognostic factor according to most authors is an early diagnosis (between 12 and 24 hours from the appearance of the fistula until its diagnosis) [1]. Currently the preferred method for the diagnosis of esophageal fistula is the performance of thoraco-abdomino CT scan with oral contrast because it has been shown to be more sensitive than esophagogastric transit. The use of upper digestive endoscopy has also shown greater sensitivity than transit but represents a more invasive diagnostic method and does not allow for the presence of collections or mediastinal involvement $[1,7]$. A dehiscence of the anastomosis is a challenge for the surgeon. Among the surgical options, repair of the anastomosis (in the absence of sepsis and small defects), section and re-make a new anastomosis or an esophageal diversion have been described. The last option considerably increases morbidity, delaying enteral feeding and requiring a second intervention to restore intestinal transit [9]. In recent years, several methods have been described for the treatment of esophago-jejunal fistula, avoiding surgical intervention. Devices such as E-VAC (intraluminal or intracavitary endoscopic vaccum therapy), the Over-the-ScopeClip (OTSC) or the submucosa injection of fibrin have demonstrated their effectiveness in closing the esophageal fistula $[1,6,10]$.

On the other hand, non-operative treatment, consisting of absolute diet, parenteral nutrition, antibiotic therapy and drainage of collections has proven useful in patients with small defects in the absence of sepsis.Self- expandable metal stents were first used in the early 90's in cases of obstruction or perforation as palliative treatment in patients with esophageal cancer [11]. Since then, the use of stents has extended to cases of esophageal perforation after antireflux surgery, myotomy or diverticulectomy, as described by Freeman in his series of 21 patients treated by stents with $95.3 \%$ success [9] . Other authors, such as Schubert, successfully describe the management of esophageal fistulas at the thoracic level in the immediate postoperative period [12]. However, its use is not a new concept. Symonds in the early nineteenth century describes the successful treatment of a pregnant woman by placing a rigid tube in the esophagus that presented dysphagia secondary to an esophageal carcinoma [13]. Mousseau and Celestin first and Atkinson and Ferguson later, developed the different devices that we know today [9].

Actually, there are different types of esophageal stents; metallic, plastic or coated. Metals and plastics do not adhere sufficiently to the esophageal surface compared to coated stents, and their migration rate is higher [10]. Despite this, some authors do not find differences between partially coated stents versus metal or plastic stents [14]. Among its advantages, it is described that they reduce morbidity and mortality, allow early initiation of nutrition through the enteral route and reduce hospital stay. However, among its disadvantages, is its migration rate (13-46\%, although it decreases with fixation by means of endoclips or self-suturing mechanisms), bleeding, perforation or stricture [2]. Wong describes the start of enteral feeding in his series after checking the absence of stent migration at 24 hours [2]. Most authors agree to remove the stent after 4-6 weeks from its placement. The size of the defect (less than $20 \mathrm{~mm}$ ) or a defect less than $70 \%$ of the circumference are the main factors related to the closure of the defect [1]. In our case, being a defect of approximately $80 \%$ (the largest known after review of the literature successfully treated) it was decided to remove the prosthesis after 8 weeks.

\section{Conclusion}

In conclusion, nowadays the curative management of gastric cancer is fundamentally surgical. The leakage of the esophagojejunal anastomosis during the postoperative period of a radical gastrectomy considerably increases the morbidity and mortality of the patient. The use of esophageal stents in selected patients avoids additional surgeries and decreases the morbidity of the process with excellent results.

\section{References}

1. Gong W, Li J (2017) Combat with esophagojejunal anastomotic leakage after total gastrectomy for gastric cancer: A critical review of the literature. Int J Surg 47: 18-24.

2. Choi CW, Kang DH, Kim HW, Park SB, Kim SJ, et al. (2017) Full covered self expandable metal stents for the treatment of anastomotic leak using a silk thread. Medicine (Baltimore) 96(29): e7439.

3. Oshi M, Kunisaki C, Miyamoto H, Kosaka T, Akiyama H, et al. (2018) Risk Factors for Anastomotic Leakage of Esophagojejunostomy after Laparoscopy-Assisted Total Gastrectomy for Gastric Cancer. Dig Surg 35(1): 28-34.

4. Hummel R, Bausch D (2017) Anastomotic Leakage after Upper Gastrointestinal Surgery: Surgical Treatment. Visc Med 33(3): 207-211. 
5. Lee SR, Kim HO, Park JH, Yoo CH (2018) Clinical Outcomes of Endoscopic Metal Stent Placement for Esophagojejunostomy LeakageAfter Total Gastrectomy for Gastric Adenocarcinoma. Surg Laparosc Endosc Percutan Tech 28(2): 113-117.

6. Turkyilmaz A, Eroglu A, Aydin Y, Tekinbas C, Muharrem Erol M, et al. (2009) The management of esophagogastric anastomotic leak after esophagectomy for esophageal carcinoma. Dis Esophagus 22(2): 119126.

7. Aday U, Gündeş E, Çiyiltepe H, Çetin DA, Gülmez S, et al. (2017) Examination of anastomotic leak with aqueous contrast swallow after total gastrectomy: Should it be carried out routinely? Contemp Oncol (Pozn) 21(3): 224-227.

8. Persson S, Rouvelas I, Kumagai K, Song H, Lindblad M, et al. (2016) Treatment of esophageal anastomotic leakage with self-expanding metal stents: analysis of risk factors for treatment failure. Endosc Int Open 4(4): E420-E426.

9. Freeman RK, Ascioti AJ, Wozniak TC (2007) Mpostoperative esophageal leak management with the Polyflex esophageal stent. J Thorac Cardiovasc Surg 133(2): 333-338.

\section{ISSN: 2574-1241}

DOI: 10.26717/BJSTR.2018.06.001391

Fernando Mendoza-Moreno. Biomed J Sci \& Tech Res

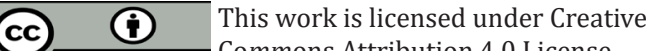

Submission Link: https://biomedres.us/submit-manuscript.php
10. Raimondo D, Sinagra E, Facella T, Rossi F, Messina M, et al. (2014) Self-expandable metal stent placement for closure of a leak after total gastrectomy for gastric cancer: report on three cases and review of the literature. Case Rep Gastrointest Med 2014:409283

11. Bektaş H, Gürbulak B, Düzköylü Y, Çolak Ş, Gürbulak EK, et al. (2017) Clinical Outcomes of Upper Gastrointestinal Stents and Review of Current Literature. JSLS 21(4): e2017.00058.

12. Schubert D, Scheidbach H, Kuhn R, Wex C, Weiss G, et al. (2005) Endoscopic treatment of thoracic esophageal anastomotic leaks by using silicone-covered, self-expanding polyester stents. Gastrointest Endosc 61(7): 891-896.

13. Symonds CJ (1887) The treatment of malignant stricture of the oesophagus by tubage or permanent catheterism. Br Med J 23 (1): 870871.

14. Sousa P, Castanheira A, Martins D, Pinho J, Araújo R, et al. (2017) Treatment of Postoperative Leaks of the Upper Gastrointestinal Tract with Colonic Self-Expandable Metal Stents. GE Port J Gastroenterol 24(4): 169-175

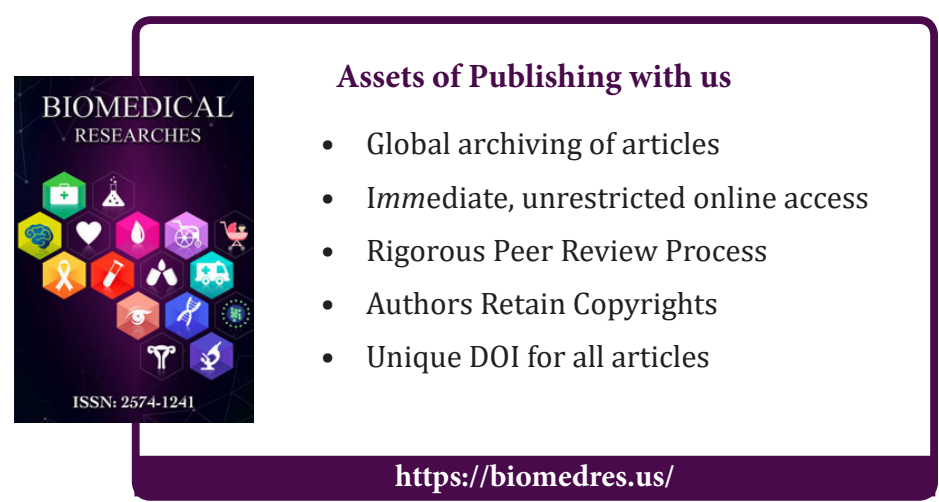

